EPiC Series in Computing
Volume 73, 2020, Pages 516-516
LPAR23. LPAR-23: 23rd International
Conference on Logic for Programming,
Artificial Intelligence and Reasoning

\title{
Models of Concurrent Kleene Algebra
}

\author{
Alexandra Silva \\ University College London, UK \\ alexandra.silva@gmail.com
}

\begin{abstract}
Kleene Algebra and variants thereof have been successfully used in verification of sequential programs. The leap to concurrent programs offers many challenges, both in terms of devising the right foundations to study concurrent variants of Kleene Algebra but also in finding the right models to enable effective verification of relevant programs. In this talk, we will review existing and ongoing work on concurrent Kleene Algebra with a focus on a variant called partially observable concurrent Kleene algebra (POCKA). POCKA offers an algebraic framework to reason about concurrent programs with control structures, such as conditionals and loops. We will show how a previously developed technique for completeness of Kleene Algebra can be lifted to prove that POCKA is a sound and complete axiomatization of a model of partial observations. We illustrate the use of the framework in the analysis of sequential consistency, i.e., whether programs behave as if memory accesses taking place were interleaved and executed sequentially.

The work described in this invited talk is based on [1, 2, 3], and it is joint with a wonderful group of people: Paul Brunet, Simon Docherty, Tobias Kappé, Jurriaan Rot, Jana Wagemaker, and Fabio Zanasi.
\end{abstract}

\section{References}

[1] Tobias Kappé, Paul Brunet, Jurriaan Rot, Alexandra Silva, Jana Wagemaker, and Fabio Zanasi. Kleene algebra with observations. In Wan Fokkink and Rob van Glabbeek, editors, 30th International Conference on Concurrency Theory, CONCUR 2019, volume 140 of LIPIcs, pages 41:1-41:16. Schloss Dagstuhl - Leibniz-Zentrum für Informatik, 2019.

[2] Tobias Kappé, Paul Brunet, Alexandra Silva, Jana Wagemaker, and Fabio Zanasi. Concurrent Kleene algebra with observations: from hypotheses to completeness. In Jean Goubault-Larrecq and Barbara König, editors, 23rd International Conference on Foundations of Software Science and Computation Structures, FOSSaCS 2020, LNCS. Springer, 2020.

[3] Jana Wagemaker, Paul Brunet, Simon Docherty, Tobias Kappé, Jurriaan Rot, and Alexandra Silva. Partially observable concurrent Kleene algebra. 2020. Under Submission, April 2020. 